\title{
Speckle Noise Reduction by Fiber Scrambling for Improving the Measurement Precision of an Airborne Wind Lidar System
}

\author{
Oliver Lux, Christian Lemmerz, Fabian Weiler, Uwe Marksteiner, Benjamin Witschas, Engelbert Nagel, Oliver Reitebuch \\ German Aerospace Center (Deutsches Zentrum für Luft- und Raumfahrt, DLR), Institute of Atmospheric Physics, Oberpfaffenhofen 82234, Germany
}

Optical guidance of coherent light in multimode fibers involves the generation of a speckle pattern at the fiber output due to the interference of the modes traveling in the fiber. Since the speckle pattern is sensitive to external perturbations (temperature, stress) as well as polarization and wavelength changes of the guided light, speckle noise is introduced limiting the quality and precision of fiber-coupled devices employed in various spectroscopic and imaging applications such as optical coherence tomography or differential absorption lidar [1].

Speckle noise was also identified as one of the major contributors to the random error of an airborne directdetection Doppler wind lidar [2] which is a key instrument for the validation of the first spaceborne wind lidar Aeolus providing vertical wind profiles of the Earth. It is composed of a frequency-stabilized laser transmitter, a Cassegrain telescope and a dual-channel receiver for analyzing atmospheric backscatter signals from both molecules (Rayleigh channel) and particles (Mie channel). Precise measurement of the transmitted laser frequency and calibration of the frequency-dependent transmission of the receiver spectrometers are prerequisite for accurate wind retrieval. Hence, a small portion of the pulsed laser radiation, referred to as internal reference, is coupled into a multimode fiber via an integrating sphere and co-aligned with the free-path atmospheric signal before entering the spectrometers. The use of a multimode fiber ensures sufficient signal intensity of the internal reference, but creates a speckle pattern consisting of only about $2 \cdot 10^{3}$ speckles incident on the Rayleigh and Mie spectrometers which are based on a Fabry-Pérot and a Fizeau interferometer, respectively.

Although the speckle pattern is static over short time scales of a few seconds, slow changes in the intensity distribution of the fiber-coupled internal reference signal are caused by variations in ambient temperature and pressure acting on the fiber and the integrating sphere, which in turn individually modify the spectral responses of the illuminated Mie and Rayleigh channels. The laser polarization and frequency are stable parameters [3] and of minor influence, but in airborne operation the vibrations on the platform additionally contribute to random intensity modulations. Since the internal reference response forms the basis for the determination of the Doppler frequency shift, and thus the derived wind speed in all atmospheric range gates, the speckle-induced fluctuations significantly increase the random error of the entire wind profile. Comparison of the internal reference frequencies derived from the Rayleigh and Mie channel responses against the frequencies measured using a wavemeter [3] showed random variations on the order of 5.2 MHz (Mie) and 9.0 MHz (Rayleigh) on time scales relevant for the wind retrieval, corresponding to wind errors of $0.9 \mathrm{~m} / \mathrm{s}$ and $1.6 \mathrm{~m} / \mathrm{s}$, respectively (Fig. 1a).

Significant reduction of the internal reference response fluctuations has been accomplished by integrating a fiber scrambler in front of the multimode fiber. The mode scrambler (GiGa Concept Inc.) is based on two counterrotating step motors which squeeze a portion of a fiber at a time constant of a few milliseconds. Compressional and torsional stress of the fiber gives rise to local and stress-induced birefringence during these motions. This leads to a decorrelation of the modes supported by the fiber, thus homogenizing the intensity distribution at the fiber output and diminishing speckle noise. As a result, the response variations of the internal reference signal are lowered to $1.1 \mathrm{MHz}$ (Mie) and 4.2 MHz (Rayleigh), which corresponds to a reduction in random wind error by a factor of about five $(0.2 \mathrm{~m} / \mathrm{s})$ and two $(0.8 \mathrm{~m} / \mathrm{s})$, respectively (Fig. 1b). In addition, the intensity fluctuations are decreased by $55 \%$ (Mie) and 22\% (Rayleigh). Smoothing of the speckle pattern hence has a large impact on the measurement precision of the wind lidar and demonstrates the potential of fiber scramblers for improving the performance of fiber-coupled spectroscopic and imaging instruments suffering from speckle noise in general.
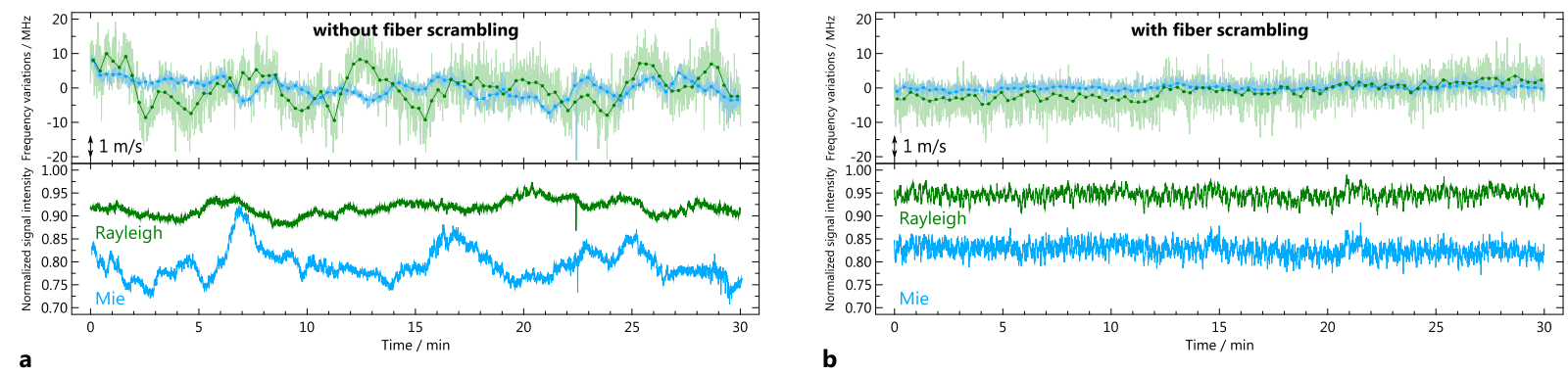

Fig. 1 Spectrometer response (top) and signal intensity (bottom) of the Doppler wind lidar internal reference without (a) and with (b) implementation of a fiber scrambler. The data is given as mean over 20 laser pulses (solid lines) and over 700 pulses (dots).

\section{References}

[1] A. Fix, M. Quatrevalet, A. Amediek, and M. Wirth, "Energy calibration of integrated path differential absorption lidars, “ Appl. Opt. 57 (26), 7501 (2018). [2] O. Lux, C. Lemmerz, F. Weiler, U. Marksteiner, B. Witschas, S. Rahm, A. Schäfler, and O. Reitebuch, "Airborne wind lidar observations over the North Atlantic in 2016 for the pre-launch validation of the satellite mission Aeolus, " Atmos. Meas. Tech. 11 (6), 3297 (2018).

[3] C. Lemmerz, O. Lux, O. Reitebuch, B. Witschas, and C. Wührer, "Frequency and timing stability of an airborne injection-seeded Nd:YAG laser system for direct-detection wind lidar," Appl. Opt. 56 (32), 9057 (2017). 